\title{
Neugestaltung und Optimierung der Lagerlogistik der Funkwerk Dabendorf GmbH
}

\author{
Sven Landvoigt, Lutz Pfister, Herbert Sonntag
}

\section{Einleitung}

\subsection{Projektaufgabe}

Die Projektaufgabenstellung umfasste, ein Lagerhallenlayout für einen gegebenen Hallenbaukörper, mit einer Länge von $50 \mathrm{~m}$, einer Breite von $40 \mathrm{~m}$ und einer nutzbaren Höhe von 7,5 m, zu erstellen. Unter Zugrundelegung eines klaren logistischen Konzeptes soll versucht werden, effiziente Lagerkapazitäten zu schaffen, die Raumhöhe maximal auszunutzen und die gesetzlichen Vorschriften zu berücksichtigen. Folgende Bereiche sollen in die Halle integriert werden:

- Wareneingang

- Wareneingangsprüfung

- Lagerung und Kommissionierung von Material

- Verpackung von Fertigprodukten

- Warenausgangsprüfung

- Warenausgang/Versand.

Für das Lager sollte ein Ausrüstungsvorschlag erstellt und auf die logistischen Abläufe der Funkwerk Dabendorf $\mathrm{GmbH}$ abgestimmt werden. Zu berücksichtigen waren hierbei die Zwänge, die durch die Zusammenarbeit mit der Automobilindustrie entstehen.

\subsection{Unternehmensbeschreibung der Funkwerk Dabendorf GmbH}

Die Funkwerk Dabendorf GmbH entwickelt und fertigt mobile Kommunikationslösungen für den Einsatz in Fahrzeugen. Aufgrund des umfangreichen Know-how in der Hochfrequenztechnik und der langjährigen Erfahrungen in elektrischem und mechanischem Design, ist das Funkwerk Dabendorf zu einem gefragten Partner von Automobilherstellern geworden. Zu den Partnern gehören z. B. Audi, BMW, DaimlerChrysler und Landrover. Je nach Kundenanforderungen wird speziell entwickelt und produziert. Das Funkwerk Dabendorf ist OEM- Lieferant der Automobilindustrie und nach TS 16949 zertifiziert. Mit der Compenser®-Technologie, einer speziell für Mobilfunkanwendungen im Fahrzeug entwickelten Verstärkertechnik, ist das Funkwerk Dabendorf weltweit führend. Durch den Compenser® (siehe Abb. 1) werden sämtliche HF-Verluste im Signalweg zwischen Handy und Außenantenne ausgeglichen und die Verbindungsstabilität der Mobilfunkverbindung im Fahrzeug gewährleistet.

Neben der Belieferung der Automobilindustrie ist die Funkwerk Dabendorf GmbH auch Marktführer im Nachrüstmarkt.

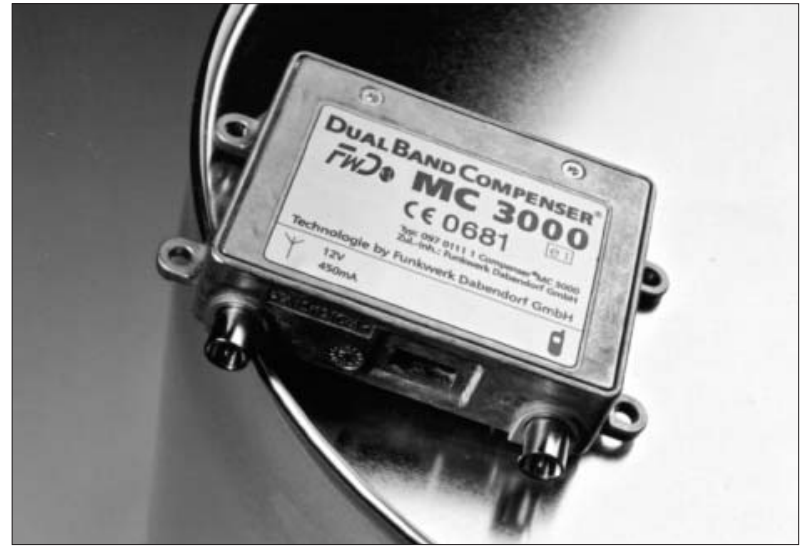

Abbildung 1: Compenser® [1]

\section{Ausgangssituation vor der Neuplanung}

Die logistische Organisation der vorhandenen Bereiche sowie die Lagerung von Waren sind Ausgangspunkt und Voraussetzung für die Neugestaltung und Optimierung der Lagerlogistik der Funkwerk Dabendorf GmbH.

\subsection{Flächenaufteilung der einzelnen Bereiche in der alten Halle}

Folgende Bereiche sind in der alten Halle vorhanden:

- Wareneingang

- Wareneingangsprüfung

- Lagerung und Kommissionierung von Material (MLI, Lager, Kommissionierbereich)

- Verpackung von Fertigprodukten

- Warenausgangskontrolle (Vor- und Nachkontrolle)

- Warenausgang/Versand der Ware.

Da die vorhandenen Kapazitäten in der alten Halle nicht ausreichen, wird eine Großzahl von Flächen von mehreren Bereichen genutzt und zusätzlich Flächen im Nebengebäude. Dies hat zur Folge, dass lange und zeitaufwendige Transportwege entstehen.

Die Gesamtfläche beträgt ca. $1075 \mathrm{~m}^{2}$ (siehe Abb. 2).

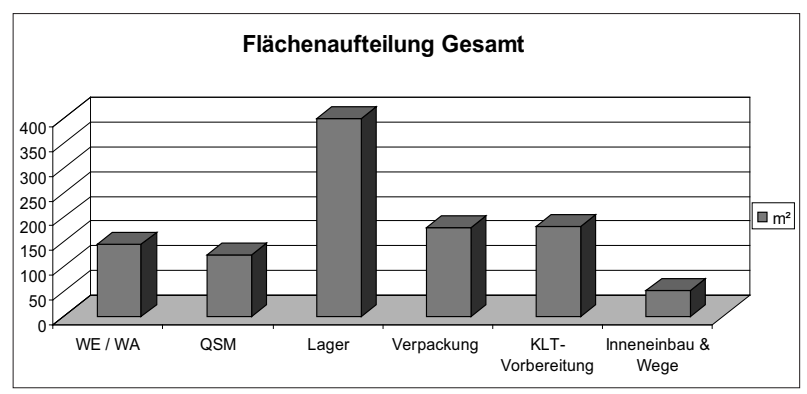

Abbildung 2: Flächenaufteilung Gesamt 


\subsection{Lagerorganisation - Bestandssituation}

Im Lager wird das Prinzip der chaotischen Lagerhaltung aus Gründen der Platz- und Raumersparnis; das FIFOVerfahren wegen der Schnelllebigkeit der Produkte und Chargen angewandt. Es sind keine festen Lagerplätze für die Materialien festgelegt - alles kann überall lagern. Eine Ausnahme bilden Rollenmaterial und bestimmte Artikel, die nur im Paternoster (wegen Form, Größe, Staub usw.) eingelagert werden können.

Die Materialbewegungen im Lager erfolgen entweder manuell oder mittels Handhubwagen und Deichselstapler. Im Materiallager werden gelagert:

- elektrische Bauelemente

- DIN und Normteile

- Zeichnungsteile

- Vorserienteile.

Die Auswertung einer ABC- Analyse (Stand Juli 2004) hat folgendes ergeben:

- A-Artikel - 149 Stück

- B-Artikel - 434 Stück

- C-Artikel - 3719 Stück.

Insgesamt lagern 10130 Artikel auf Lagerplätzen. Die Gewichte pro Entnahmeeinheit sind aufgrund der Artikelstruktur sehr unterschiedlich, sie können zwischen 0,1 $\mathrm{kg}$ und $20 \mathrm{~kg}$ variieren.

Im Lager wird die einstufige bzw. auftragsbezogene Kommissionierung angewandt. Der Auftrag wird in einem Arbeitsprozess zusammengestellt. Der Kommissionierer entnimmt nur für einen Auftrag, Position für Position, die verschiedenen Artikelmengen, übergibt den kompletten Auftrag an die Sammelstelle, bucht den Auftrag. Anschließend beginnt der Kommissionierer mit der Kommissionierung des nächsten Auftrages. Hierbei wird einzonig gearbeitet.

In den Weitspann-/Fachbodenregalen und Palettenregalen wird das Prinzip „Mann zur Ware“ angewandt, der Kommissionierer bewegt sich im Lager eindimensional, dass heißt zu Fuß. Die Entnahme der Teile in den Weitspannund Fachbodenregalen erfolgt manuell. In den Palettenregalen erfolgt die Entnahme mechanisch - mit Hilfe eines Deichselstaplers. Die entnommenen Artikel werden auf einen $\mathrm{C}+\mathrm{C}$ Wagen abgelegt. Dabei werden mehrere Behälter übereinander gestapelt. Bei Teilen, die im Paternoster gelagert werden, findet das System „Ware zum Mann“ Anwendung. Die Übergabe des Kommissionierbehälters oder einer Palette als artikelunreine Ladeeinheit erfolgt an einem zentralen Platz, auf der Kommissionier-/Bereitstellfläche.

Das größte Problem der alten Logistikhalle ist der Platzmangel. Er beginnt schon beim Wareneingang und zieht sich durch alle übrigen Bereiche. Jede Abteilung muss sich auf engstem Raum bewegen und arbeiten. Aufgrund des Platzmangels werden die Verkehrswege und eigentliche Freiflächen als Arbeits- oder Abstellfläche mitbenutzt, wodurch die Übersichtlichkeit verloren geht.

\section{Neugestaltung von Logistikhalle und Beschaffungslager}

Die äußeren Abmessungen der neuen Halle betragen $50 \mathrm{~m}$ x 40 m (Grundfläche 2000 m²$^{2}$ ). Die Höhe der Halle beträgt an den Außenseiten 6,5 m und in der Hallenmitte $10 \mathrm{~m}$. In der Halle befindet sich ein 2-geschossiger Einbau. Die vorhandenen Bereiche wurden in die neue Logistikhalle integriert. Über dem Verpackungsbereich wird eine Bühne mit den Abmessungen von ca. $22 \mathrm{~m} \mathrm{x} 10$ m eingezogen. Die Raum- bzw. Lagerfläche wird ohne Veränderung des Bauwerkes durch die zusätzliche Bühnenebene vergrößert und die Raumhöhe optimaler ausgenutzt. Es ist gleichzeitiges lagern und arbeiten möglich.

\subsection{Innerbetrieblicher Transport und Lademittel}

Der innerbetriebliche Transport wird mittels Transportarbeiter (manuelles Transportsystem) durchgeführt. Er kann sich je nach Art und Umfang des Transportgutes den nachfolgenden Techniken (Hilfsmittel) bedienen:

- Deichselstapler

- Schubmaststapler

- Handhubwagen

- Tischwagen

- C+C Wagen

- Tablettwagen

- Scheren- Rollen-/Röllchenbahnen

- Etagenwagen.

Da in der Funkwerk Dabendorf GmbH ein hohes Maß an Flexibilität und die Freihaltung der Verkehrswege von fest installierten Einrichtungen gefordert wurde, kommen gleislose Flurförderzeuge zum Einsatz. Eingesetzt werden sie im innerbetrieblichen Transport für unregelmäßige anfallende Transport- und Hubarbeiten. Außerdem ist der Investitionsaufwand im Verhältnis zu anderen Fördermitteln viel niedriger. Da der Transportweg in der neuen Logistikhalle meistens nicht mehr als $25 \mathrm{~m}$ lang ist, keine Steigungen enthält und der Transport nicht ständig durchgeführt wird, ist der Handbetrieb angebracht. Der Handhubwagen für den Paletten- und Behältertransport lässt sich sinnvoll und wirtschaftlich einsetzen, da:

- geringe Massen zu transportieren sind

- beengte Platzverhältnisse vorliegen

- Transportwege kurz sind (z. B. zwischen benachbarten Arbeitsplätzen)

- Transportfrequenz niedrig ist.

Mit dem Handgabelhubwagen wird der Transport von Paletten zwischen den Arbeitsplätzen vollzogen. Für die Ein- und Auslagerung der Paletten im Lager wird der Schubmaststapler eingesetzt, da er flexibel einzusetzen ist.

Für den Transport von Kartons und Rollenware zum Lagerplatz werden Etagenwagen benutzt. Der Etagenwagen besitzt mehrere Einlegeböden die man nach den eigenen Bedürfnissen verstellen kann. Mit diesem Etagenwagen ist man flexibel und die weiten Wege lassen sich gut überbrücken. Die Waren können auf die verschiedenen Etagen verteilt werden und das Übereinanderstapeln wird somit vermieden. $\mathrm{Zu}$ Beschädigungen kann es dadurch nicht mehr kommen. Außerdem werden die Etagenwagen für die Kommissionierung verwendet.

\subsection{Gestaltung des Lagers}

Im Lagerbereich befinden sich folgende Abteilungen:

- MLI

- Lager

- Kommissionierung. 


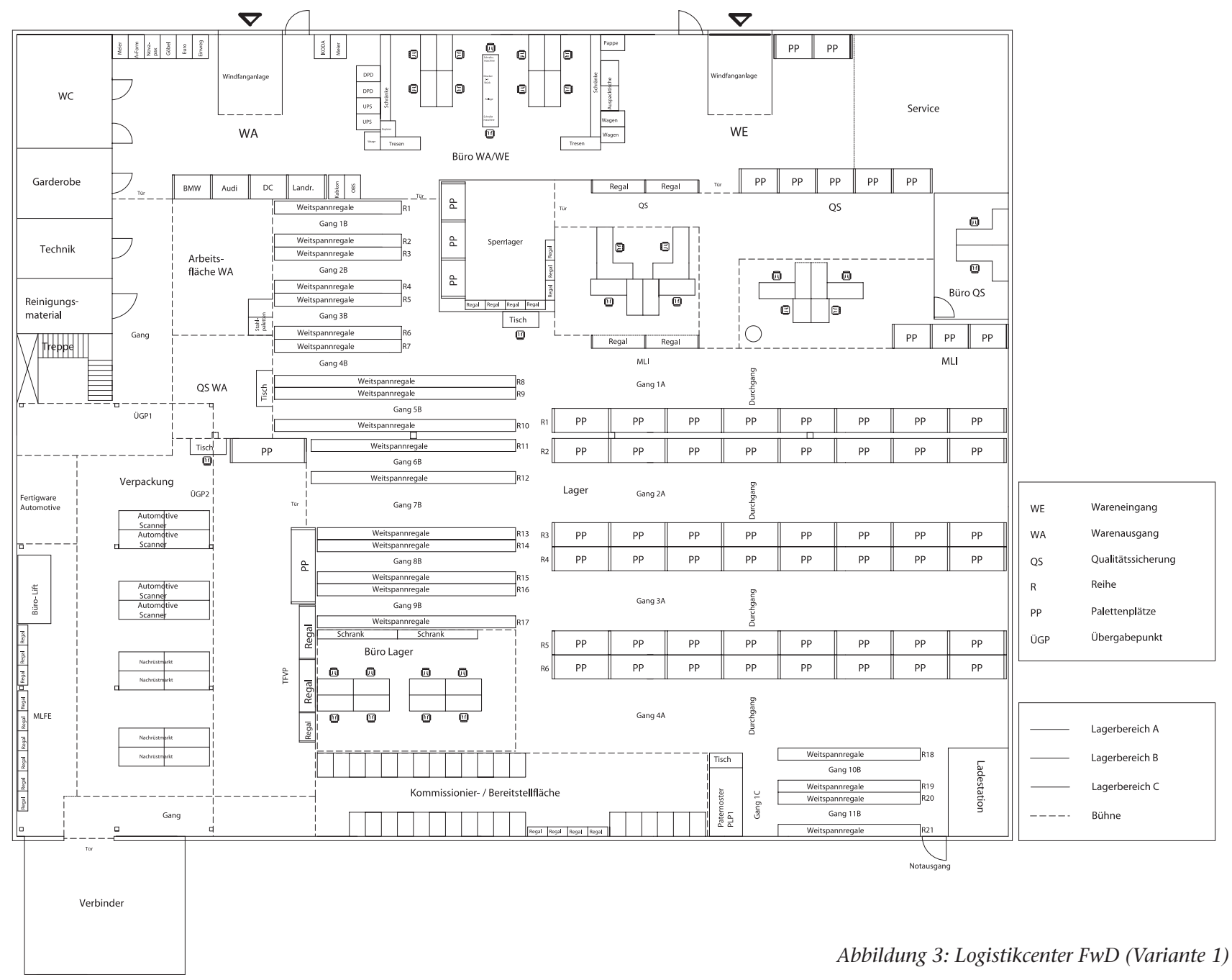

1 Gestaltung des Lagerbereiches A mit Palettenregalen Die Ausgangsbasis ist:

- Einlagerung von DIN-Flachpaletten

- Grundmaß: $1.200 \mathrm{~mm}$ x $800 \mathrm{~mm}$

- Längseinlagerung

- 5, 3 Paletten übereinander

- 3 Paletten nebeneinander (2.700 mm lichte Weite Mehrplatzlagerung).

Der Lagerbereich A (siehe Abb. 3) besteht aus 3 Doppelregalreihen (Reihe 1 - 6) mit einer Regalhöhe von $6.975 \mathrm{~mm}$. Die Gesamtkapazität beträgt 684 Stellplätze (pro Reihe 114 Stellplätze). Es sind folgende Eigenschaften zu benennen:

- 684 Stellplätze im Palettenlager

- Volumen beträgt ca. $1106 \mathrm{~m}^{3}$

- sehr guter Zugriff auf alle Artikel

- mechanisier- und automatisierbar

- sehr guter Nutzungs- beziehungsweise Füllungsgrad durch freie Platzzuordnung

- gute Einhaltung von FIFO

- gute Kommissionierleistung bei geeignetem Fördersystem

- einfache und ausbaufähige Organisation mit DV möglich

- flexibel; Ladeeinheit kann in Höhe und Breite variieren; Höhe der Feldebenen veränderbar; die größere lichte Feldbreite kann unterschiedliche Breiten von Ladeeinheiten aufnehmen
- gute Flächennutzung, da relativ wenig Ständerrahmen

- anpassungsfähig an geänderte Sortimentsstruktur

- gute Raumausnutzung, da Raumhöhe optimal ausgenutzt wird

2 Gestaltung des Lagerbereiches B (Variante 1 - 3)

In der Variante 1 wird der Lagerbereich B (siehe Abb. 3) mit „Weitspannregalen“ ausgestattet. Die Weitspannregale sind in 6 Ebenen unterteilt. Die Höhen der einzelnen Ebenen sind wie folgt festgelegt:

- Ebene 1-5 = $350 \mathrm{~mm}$

- Ebene $6=240 \mathrm{~mm}$.

Es ergeben sich die folgenden Eigenschaften:

- Lagerfläche beträgt ca. $618 \mathrm{~m}^{2}$

- Volumen beträgt $258 \mathrm{~m}^{3}$

- sehr guter Zugriff zu jedem Artikel

- relativ geringe Investitionskosten

- flexibel bei Strukturänderung, da einfach umrüstbar

- mechanisier- und automatisierbar, somit auch für hohe Umschlagsleistung möglich

- gute Ordnungsmöglichkeit und Kontrolle der Bestände

- Kommissionieren gut möglich

- einfache Organisation möglich, ausbaufähig für unterschiedliche Lagerstrategien.

In der Variante 2 wird der Lagerbereich B (siehe Abb. 4) mit Paternostern ausgestattet. Bei der manuellen Kommissionierung sucht der Kommissionierer die Lagerplätze zu Fuß auf, entnimmt die Artikel manuell und hat den 


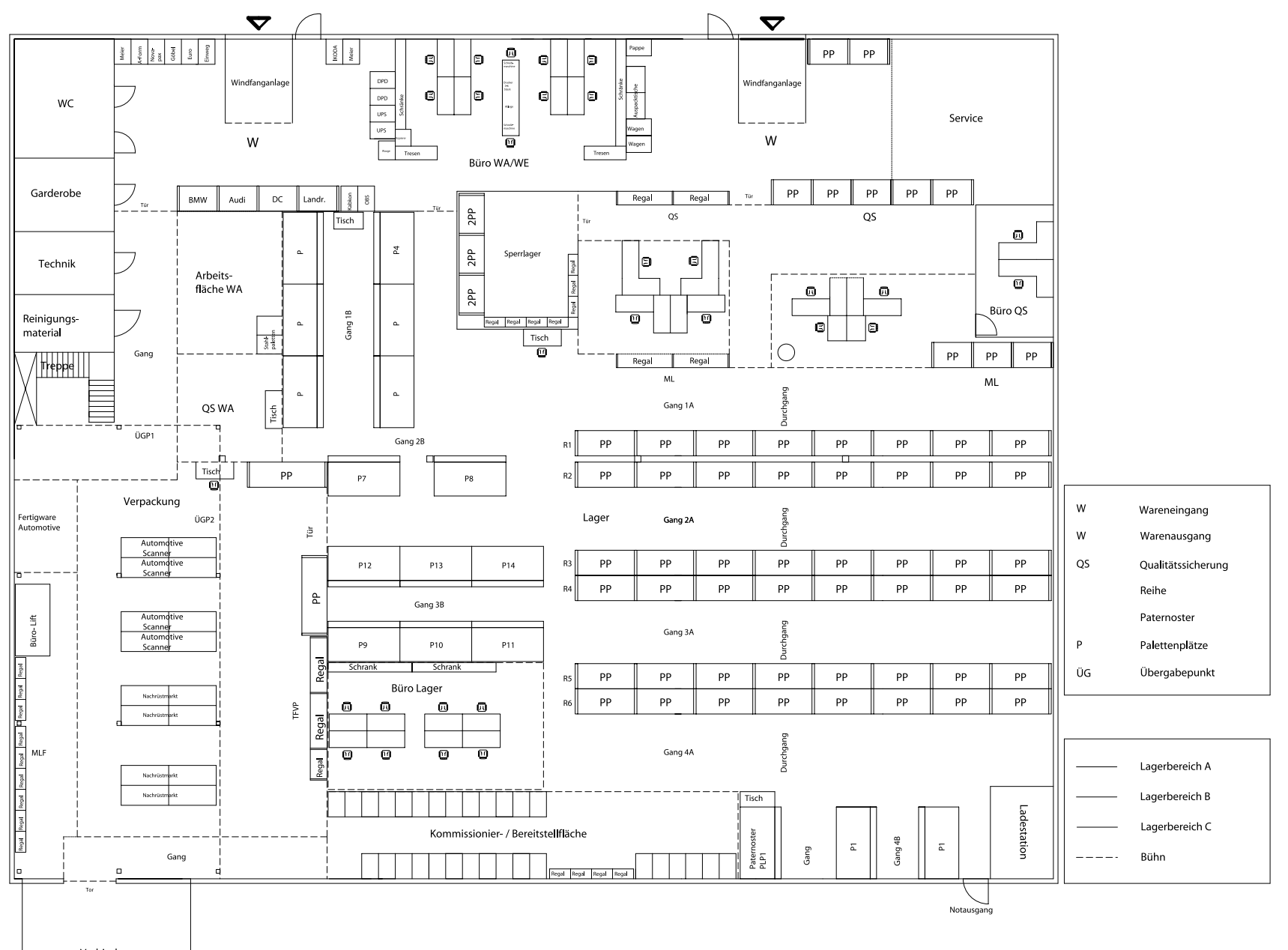

Abbildung 4: Logistikcenter FwD (Variante 2)

Auftrag kommissioniert. Um wirtschaftlich zu arbeiten, ist es erforderlich:

- Wegzeit zu reduzieren

- Greifzeit zu optimieren

- Ermüdung des Kommissionierers zu minimieren

- Kommissionierleistung zu steigern.

Um dies zu erreichen könnten die Weitspannregale durch Paternoster ersetzt werden. Durch einen Paternoster kommt es zu einer Umstellung von manuellem auf halbautomatisches Handling. Paternoster eignen sich meistens für leichte Güter, mittlere Artikelanzahl, kleine Mengen und mittlere Umschlagsleistung. Aufgrund von Tablaren ist eine variable Facheinteilung möglich. Kleinteile werden in Lagerkästen, die gleichzeitig auch als Transportkästen dienen können, untergebracht.

Es ergeben sich die folgenden Eigenschaften:

- Lagerfläche pro Paternoster beträgt ca. 62,4 m²

- optimale Nutzung der Raumhöhe

- ergonomische Greifhöhe

- abschließbar und dadurch Schutz des Gutes vor unbefugtem Zugriff

- EDV-gesteuerte Lagerhaltung

- ESD-Schutz

- Kommissionierung (Ware zum Mann)

- barcodegesteuert $\rightarrow$ Scanner an allen Arbeitsplätzen ersparen zeitaufwendige Zahleneingaben und verhindern Fehleingaben
- FIFO gut realisierbar

- freie Lagerplatzzuordnung möglich

- sehr gute Flächennutzung

- Schutz des Gutes vor Verschmutzung

- Verminderung der Unfallgefahr $\rightarrow$ kein Bücken, kein Leiternsteigen

In der Variante 3 wird der Lagerbereich B mit einer „zweigeschossigen Regalanlage“ ausgestattet. Die Ausnutzung von vorhandenem Hallenvolumen spielt eine wesentliche Rolle bei der Wirtschaftlichkeit von Anlagen. Der Flächennutzungsgrad des Gebäudes wird indirekt vervielfacht; wirtschaftlich besonders interessant, da die Regalkonstruktionen tragende Elemente für die Zwischenebenen sind. Diese mehrgeschossige Bauweise bietet den Hauptvorteil, dass die Entnahme von Lagergut durch viele Personen gleichzeitig - innerhalb kürzester Zeit - erfolgen kann.

Mit durchdachten Stahlbühnen/Regalbühnen (siehe Abb. 5) gewinnt man eine zusätzliche Lagerebene bei gleicher Grundfläche. Bühnenanlagen werden individuell für unterschiedliche Belastungen nach den speziellen Erfordernissen konzipiert. Bei Regalbühnen werden die Bühnengänge zwischen den Regalzeilen eingebaut. Der Vorteil liegt darin, dass die Fachbodenregale als Trägersysteme genutzt werden können. 


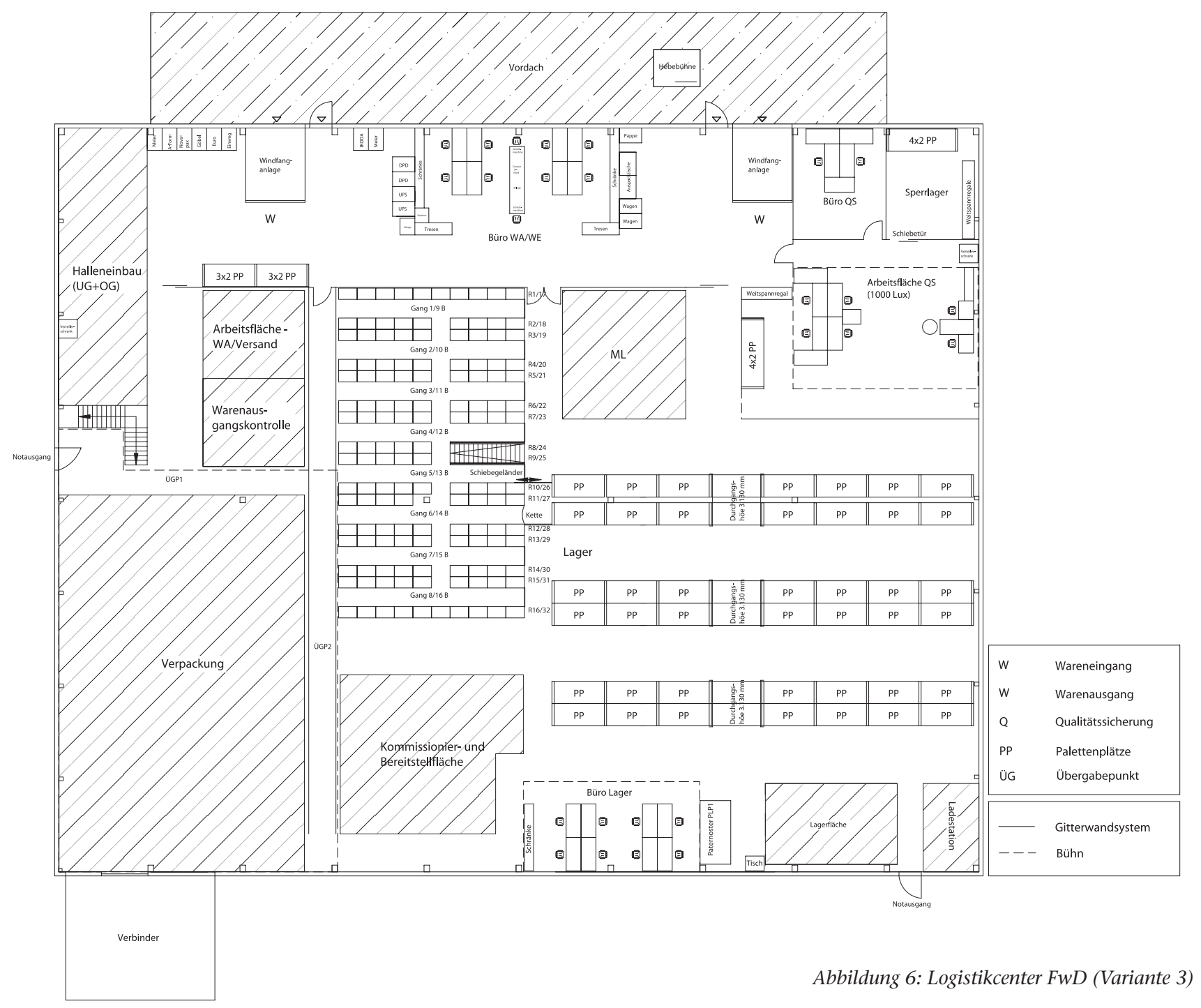

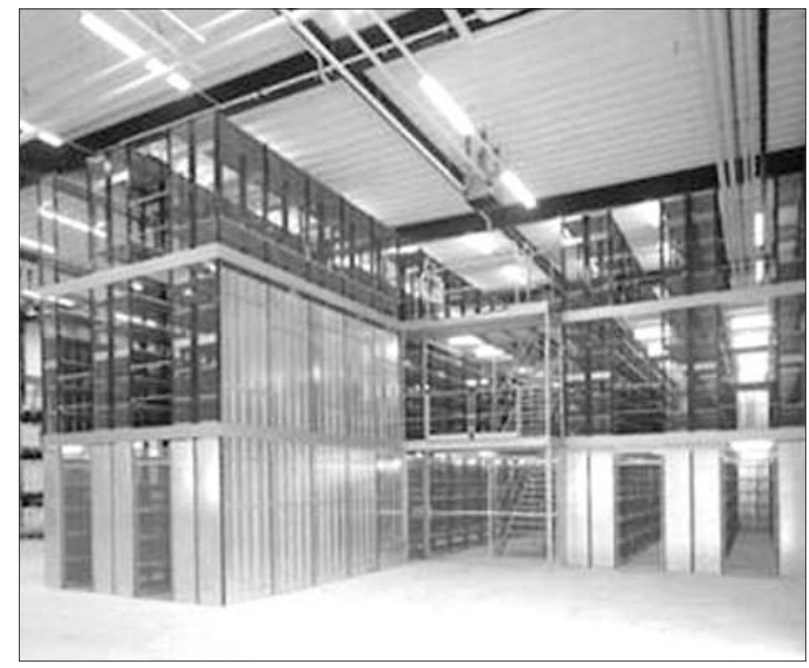

Abbildung 5: Mehrgeschossige Regalanlagen [2]

Der Lagerbereich B (siehe Abb. 6) besteht aus 32 Regalreihen (Erdgeschoss: Reihe 1-16; Obergeschoss: Reihe 1732). Die Stützrahmenhöhe liegt bei $5.500 \mathrm{~mm}$. Verwendet werden Stahlfachböden mit einer Länge von $1.000 \mathrm{~mm}$. Im Erdgeschoss befinden sich sieben Fachbodenebenen, jeweils mit einer Höhe von $350 \mathrm{~mm}$.

Die Oberkante des Laufgangbelages liegt bei $3.000 \mathrm{~mm}$.
Der Laufgangbelag ist eine Spanplatte, dessen Unterseite ist mit weißer Beschichtung ist. Im Obergeschoss befindet sich zusätzlich ein Palettenabstellplatz mit Absperrkette. An den Einfachregalen befinden sich Rückwände aus Drahtgitter. Im Obergeschoss befinden sich fünf Fachbodenebenen mit einer jeweiligen Höhe von $400 \mathrm{~mm}$.

Es sind folgenden Eigenschaften zusammenfassend $\mathrm{zu}$ benennen:

- Lagerfläche beträgt ca. $1081 \mathrm{~m}^{2}$

- Volumen beträgt 495,3 m

- gute Nutzung der Raumhöhe

- zusätzliche Lagerebene bei gleicher Grundfläche

- Kommissionieren gut möglich

- kurze Wege, da sich alle Fachbodenregale in einem Bereich befinden

- geringe Investitionskosten, da Regale gleichzeitig tragende Konstruktion für Laufebenen sind

- gute Übersicht durch kompakte Bauweise.

\subsection{Ablauf des Kommissionierens}

Kommissionierarbeiten gehören $\mathrm{zu}$ den arbeits- und personenintensivsten Tätigkeiten in der Lagerlogistik. Mehr denn je gilt hier: Zeit ist Geld! Bei der statischen Bereitstellung der Ware - nach dem Prinzip „Mann zur Ware", welches bei der Funkwerk Dabendorf GmbH 
angewandt wird, wurde darauf geachtet, dass sich die Ware stets im direkten Zugriff für den Kommissionierer befindet. Weitspann-/Fachbodenregale bieten dafür gute Voraussetzungen: flexibel im Einsatz, leicht verstellbar und beliebig kombinierbar. Die Kommissionierung per Hand wird leichter, die Zugriffszeiten verkürzen sich.

\section{Kommissionieren mit Fachboden-Regaltechnik}

Trotz aller modernen Technologien, die zunehmend im Lager- und Kommissionierbereich Einzug halten, ist das klassische Fachbodenregal (siehe Abb. 7) das am weitesten verbreitete Hilfsmittel auf diesem Gebiet. Sein Einsatz erfolgt vorzugsweise, wenn aus einem sehr breiten Artikelsortiment pro Artikel nur kleine bis mittlere Mengen bevorratet bzw. zur Kommissionierung bereitgestellt werden sollen. Ordnungsmöglichkeiten, Übersichtlichkeit und Kontrolle der Lagerbestände sind als gut einzustufen.

Fachbodenregalanlagen sind nachträglich mechanisierbar und ggf. automatisierbar; sie sind auch aufstockbar und bieten somit Potential für später erforderliche höhere Kommissionierleistungen. Die Zuteilung der Lagerplätze erfolgt in Abhängigkeit von der Umschlaghäufigkeit der Artikel, sprich Schnelldreher nahe dem Hauptkommissionierweg, Langsamdreher in entfernte Bereiche der Regalgänge. Durch die Auswahl einer geeigneten Beschriftung der Lagerplätze, ist ein schnelles und eindeutiges Erkennen garantiert. Durch die Festlegung der Kommissionierstrategie, soll jeder Auftrag für sich kommissioniert werden. Diese Maßnahmen reduzieren den systemtypischen Nachteil relativ langer Kommissionierwege.

Die Ergonomie spielt dabei eine wichtige Rolle. Eine Fachtiefe von $600 \mathrm{~mm}$ wurde ausgewählt und ausreichend breite Arbeitsgänge angelegt. Die flexiblen Stecksysteme erleichtern und beschleunigen die in jedem Lager „vorprogrammierten“ Veränderungen und Umbauten, deren Vorteile ausschließlich in der niedrigen Investition zu sehen sind. Gute Regalsysteme verfügen über umfangreiches Zubehör, mit dessen Hilfe die unterschiedlichsten Güter individuell und optimiert gelagert werden können.

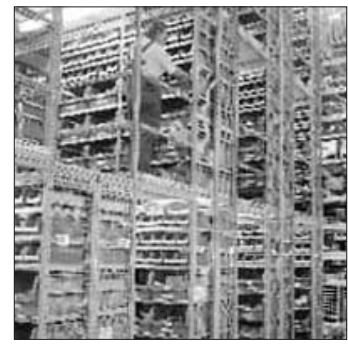

Abb. 7: Fachbodenregalanlage [3]

\section{Kommissionieren mit Paletten-Regaltechnik}

Jede Palette ist bei diesem System (siehe Abb. 8) leicht zugänglich und die Regalaufstellung kann leicht verändert und ergänzt werden. Die relativ niedrige Investition und die Flexibilität haben dieses System zu einem unverzichtbaren Baustein in der Lager- und Kommissioniertechnik gemacht. Für große Mengen je Artikel und einer mittleren bis hohen Umschlagsleistung wird dieses System gerne eingesetzt. Die Mehrplatzlagerung mit 2-4 Paletten pro Fach nebeneinander ist die gebräuchlichste Form. Die Paletten im Regal werden in Längsrichtung eingelagert. Das Stecksystem der Traversen in einem gleichmäßigen
Verstellraster ermöglicht die einfache Anpassung der Fachhöhen an wechselnde Beladehöhen der Paletten. Das Palettenregalsystem ermöglicht über die Standardtraversen hinaus mit seinen Stufentraversen den alternativen Einsatz von Gitterrostböden, Stahlfachböden oder Fachebenen aus Spanplatten, damit auch jedes mit Stapler bewegtes Lagergut sicher gelagert werden kann.

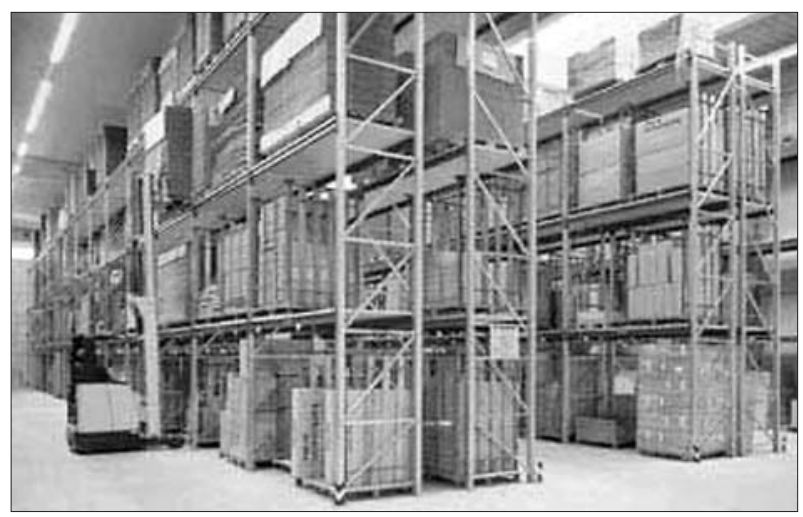

Abbildung 8: Palettenregalanlage [4]

\subsection{Gestaltung Verkehrswege und -gänge}

Es gibt 3 Nutzungsarten von Wegen:

- Gemeinsamer Geh- und Fahrverkehr

- Fahrverkehr

- Gehverkehr.

Die Lagereinrichtungen wurden nach den Richtlinien für Lagereinrichtungen und -geräte (GUV-R 1/428) aufgestellt. Die Lagereinrichtungen wurden so geplant, dass ausreichend bemessene Gänge vorhanden sind. Verkehrswege für Fußgänger zwischen Lagereinrichtungen sind mindestens 1,25 m breit. Gänge, die nur für das Be- und Entladen von Hand bestimmt sind (Nebengänge), sind mindestens $1 \mathrm{~m}$ breit.

Die Verkehrswege für den Schubmaststapler sind so breit, dass auf beiden Seiten des Fördermittels ein Sicherheitsabstand von mindestens $0,5 \mathrm{~m}$ gewährleistet ist. Da die Wege für den Fahrverkehr und zum Gehverkehr benutzt werden, sind die Randzuschläge sogar mit 0,75 m angesetzt worden. Bei der Bemessung ist auch der Platzbedarf für Rangiervorgänge berücksichtigt.

Alle Verkehrswege sind übersichtlich angelegt und verlaufen möglichst geradlinig. Alle Wege für den Fahrverkehr sind ohne Neigung angelegt worden. Die Wege für den Gehverkehr sind nicht durch einzelne Stufen unterbrochen. Da die neue Logistikhalle mehr als $1000 \mathrm{~m}^{2}$ Grundfläche hat, müssen laut DIN 18225 und den Arbeitsstätten-Richtlinien die Verkehrswege eine eindeutige Kennzeichnung bekommen. Es gibt Fußwege, Fahrwege und gemeinsame Fuß- und Fahrwege.

Die Durchfahrten der Palettenregale haben eine lichte Höhe von 3,13 m. Die lichte Höhe der Durchfahrten ist in Abhängigkeit von der Höhe des eingesetzten Fördermittels, inklusive eines Sicherheitsabstandes von $200 \mathrm{~mm}$, bemessen. Rettungswege führen auf möglichst kurzem Wege ins Freie oder zu einem gesicherten Bereich. Sie dürfen nie verstellt oder eingeengt werden. Türen müssen in Fluchtrichtung ausschlagen und sich ohne Hilfsmittel leicht öffnen lassen. Kraftbetätigte Schiebe-, Falt- und Rolltore müssen notfalls von Hand zu öffnen sein. Die Umstellung von Kraft- 
auf Handbetrieb muss unkompliziert und leicht zugänglich sein. Sicherheitsbeleuchtung für Flucht- und Rettungswege ist erforderlich, wenn bei Ausfall der Allgemeinbeleuchtung das Verlassen der Arbeitsplätze nicht gewährleistet ist.

Von besonderer Bedeutung ist die eindeutige und leicht erkennbare Kennzeichnung der Rettungswege und Notausgänge. Hierzu sind die „Rettungszeichen für Rettungswege und Notausgänge/Türen im Verlauf von Rettungswegen" (UVV - „Sicherheits- und Gesundheitsschutzkennzeichnung" VBG 125) verwendet worden. Die Rettungszeichen bestehen aus lang nachleuchtenden Materialien. Verkehrswege sind während der Dauer der Benutzung ausreichend und sachgemäß beleuchtet (DIN 5035 Teil 2). Beim Anlegen der Beleuchtungseinrichtungen wurde darauf geachtet, dass sich aus der Art der Beleuchtung keine Unfall- oder Gesundheitsgefahren für die Beschäftigten ergeben.

\subsection{Maße für die Verpackungseinheiten}

Laut dem Verpackungshandbuch der Funkwerk Dabendorf GmbH sind die neuen Maße auf max. 400 x 400 x 300 mm (L x B x H) (Ausnahme: elektronische Bauelemente als Rollenware) festgelegt. Das Gewicht darf max. $15 \mathrm{~kg}$ betragen. Die Höhe der Verpackungseinheit auf einer Euro- Palette darf jetzt max. $1.500 \mathrm{~mm}$ betragen.

In der neuen Logistikhalle sollen keine Kartons mehr übereinander gestapelt werden, sondern nur noch nebeneinander, aufgrund der Übersichtlichkeit und damit FIFO besser eingehalten und beachtet werden kann.

Aufgrund der festen Höhen der einzelnen Ebenen in den Fachboden- und Palettenregalen, müssen sich die Lieferanten an die neu definierten Maße der Verpackungseinheiten halten.

\section{Bewertung}

\subsection{Flächenvergleich zwischen alter und neuer Halle (Variante 3 - Lagerbereich B)}

Die folgende Abbildung und Tabelle soll einen Überblick über die Veränderungen zwischen alter und neuer Logistikhalle geben:

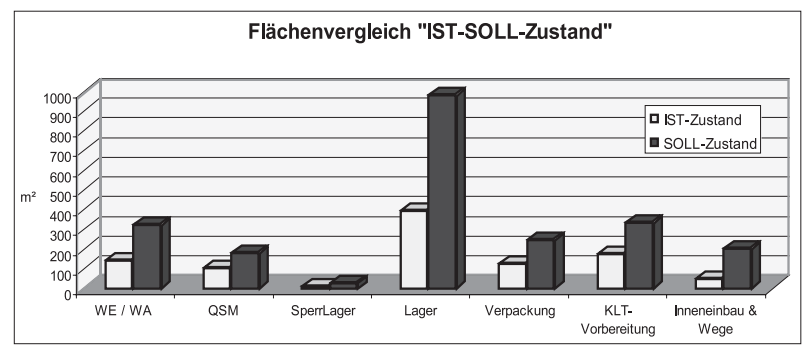

Abbildung 9: Flächenvergleich „IST-SOLL-Zustand“

\begin{tabular}{|l|l|}
\hline Bereiche & Flächenvergrößerung um \% \\
\hline WE / WA & 127 \\
\hline QSM & 67 \\
\hline SperrLager & 114 \\
\hline Lager & 148 \\
\hline Verpackung & 93 \\
\hline KLT- Vorbereitung & 87 \\
\hline Inneneinbau \& Wege & 296 \\
\hline
\end{tabular}

Tabelle 1: Flächenvergrößerung der einzelnen Bereiche

\subsection{Flächennutzungsgrad des Lagerbereiches}

Die gesamte Lagerfläche in der neuen Logistikhalle (Variante 3 - Lagerbereich B) umfasst 987 m²$^{2}$. Unter Abzug der Flächen für Lagerbüro, MLI, Kommissionier-/Bereitstellfläche und Ladestation beträgt die Lager- Bruttofläche 796,75 m². Die Lager- Nettofläche setzt sich aus der Palettenregalanlage, der zweigeschossiger Regalanlage, des Paternosters und der Bodenlagerfläche zusammen und umfasst somit 294,08 $\mathrm{m}^{2}$. Der Flächennutzungsgrad beträgt somit ca. 37 \%.

\subsection{Vergleich der drei Varianten im Lagerbereich B}

Variante 1: Lagerbereich B mit Weitspannregale

Variante 2: Lagerbereich B mit Paternoster

Variante 3: Lagerbereich B mit zweigeschossiger Fachbodenregalanlage

In der nachfolgenden Tabelle sind die drei Varianten für den Lagerbereich B aufgeführt. Bei der Variante 2 wurden nur die Werte für einen Paternoster dargestellt. Man sieht, dass der Preis pro/ $\mathrm{m}^{2}$ Lagerfläche bei der Variante $3 \mathrm{am}$ geringsten ist. Der Lagerflächenpreis des Paternosters ist gegenüber den Varianten 1 und 3 deutlich höher und zusätzlich kommen noch jährliche Wartungskosten dazu.

\begin{tabular}{|l|l|l|l|}
\hline Variante & Investitionskosten & Lagerfläche & Preis pro $\mathrm{m}^{2}$ \\
\hline 1 & ca. $30.800 €$ & $618 \mathrm{~m}^{2}$ & ca. $50 € / \mathrm{m}^{2}$ \\
\hline 2 & ca. $41.800 €$ & $62,4 \mathrm{~m}^{2}$ & ca. $670 € / \mathrm{m}^{2}$ \\
\hline 3 & ca. $43.850 €$ & $1081 \mathrm{~m}^{2}$ & ca. $41 € / \mathrm{m}^{2}$ \\
\hline
\end{tabular}

Tabelle 2: Variantenvergleich im Lagerbereich B

\subsection{Vergleich des Lagerbereiches zwischen alter Halle und neuer Halle}

\section{Palettenplätze}

684 Palettenplätze stehen in der neuen Halle zur Verfügung. Dies sind 447 Palettenplätze mehr als in der alten Halle (237 Palettenplätze). Dies entspricht einer Steigerung um $189 \%$.

Das Volumen der Palettenregalanlage (siehe Abb. 10) ist um $765 \mathrm{~m}^{3}$ (ca. $224 \%$ ) gegenüber dem IST-Zustand gestiegen.

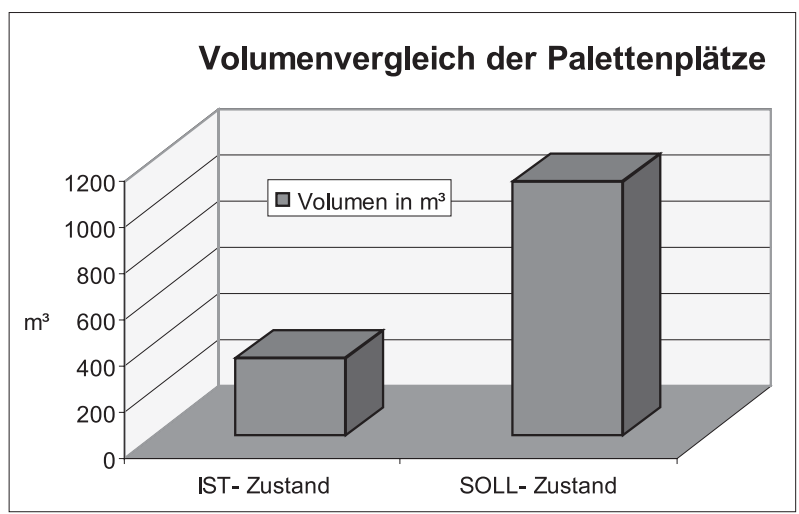

Abbildung 10: Volumenvergleich der Palettenplätze

\section{Fachboden-/Weitspannregale}

Wenn man die Lagerfläche des IST-Zustandes mit der Lagerfläche der Variante 3 vergleicht, stehen beim SOLL-Zustand $828 \mathrm{~m}^{2}$ mehr Lagerfläche zur Verfügung, dies ist eine Steigerung um ca. $327 \%$. Auf das Volumen bezogen, entspricht dies einer Steigerung um ca. 194 \% (siehe Abb. 11). 


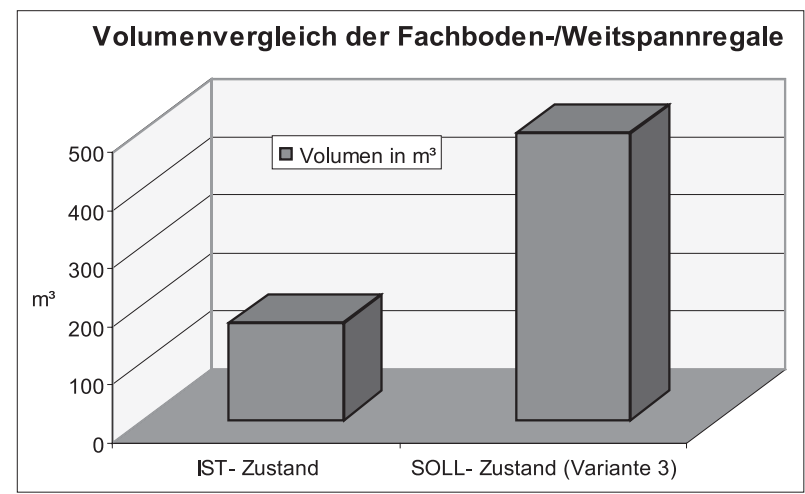

Abbildung 11: Volumenvergleich der Fachboden-/Weitspannregale

\subsection{Investitionskosten Gesamt}

\section{Lagerbereich}

In der nachfolgenden Tabelle sind die Investitionskosten für den Bereich A (Palettenregalanlage) und den Bereich B mit seinen verschiedenen Varianten dargestellt:

\begin{tabular}{|l|l|}
\hline Bereich & Investitionskosten in $€$ \\
\hline A - Palettenregalanlage & $19.200,00$ \\
\hline B - Variante 1 & $30.800,00$ \\
\hline B - Varinate 2 (pro Paternoster) & $41.800,00$ \\
\hline B - Variante 3 & $43.850,00$ \\
\hline
\end{tabular}

Tabelle 3: Investitionskosten Lagerbereich

Die Variante 1 ist zwar die mit den geringsten Investitionskosten, jedoch hat sie geringste Lagerfläche. Die Investitionskosten der Varianten 2 und 3 sind zwar höher, aber dafür ist die Lagerfläche größer. Beim Vergleich der Preise pro $\mathrm{m}^{2}$ Lagerfläche wird ganz deutlich, dass die Variante 3 letztendlich die preiswerteste ist. Die Lagerung von Ware in einem Paternoster hat zwar seine Vorteile, aber aufgrund der hohen Investitionskosten pro Paternoster gegenüber den anderen Varianten lohnt sich diese Variante der Lagerausstattung fast gar nicht. Die größten Vorteile dieser Lagerung sind zum einem, das halbautomatische Handling („Ware zum Mann“) und zum anderem die nahezu optimale Ausnutzung der Raumhöhe von 7.500 mm. Bei der Variante 1 und 3 bleibt es bei den einmaligen Investitionskosten, doch beim Paternoster kommen noch jährliche Betriebs- und Wartungskosten dazu.

\section{Investitionskosten der Neuanschaffungen}

In der nachfolgenden Abbildung sind die gesamten Investitionskosten für die Neugestaltung der Logistikhalle dargestellt. Für den Lagerbereich B wurde die Variante 3 mit in die Darstellung genommen. Das Investitionsvolumen für die Neuausstattung der Logistikhalle liegt bei ca. $168.000 €$.

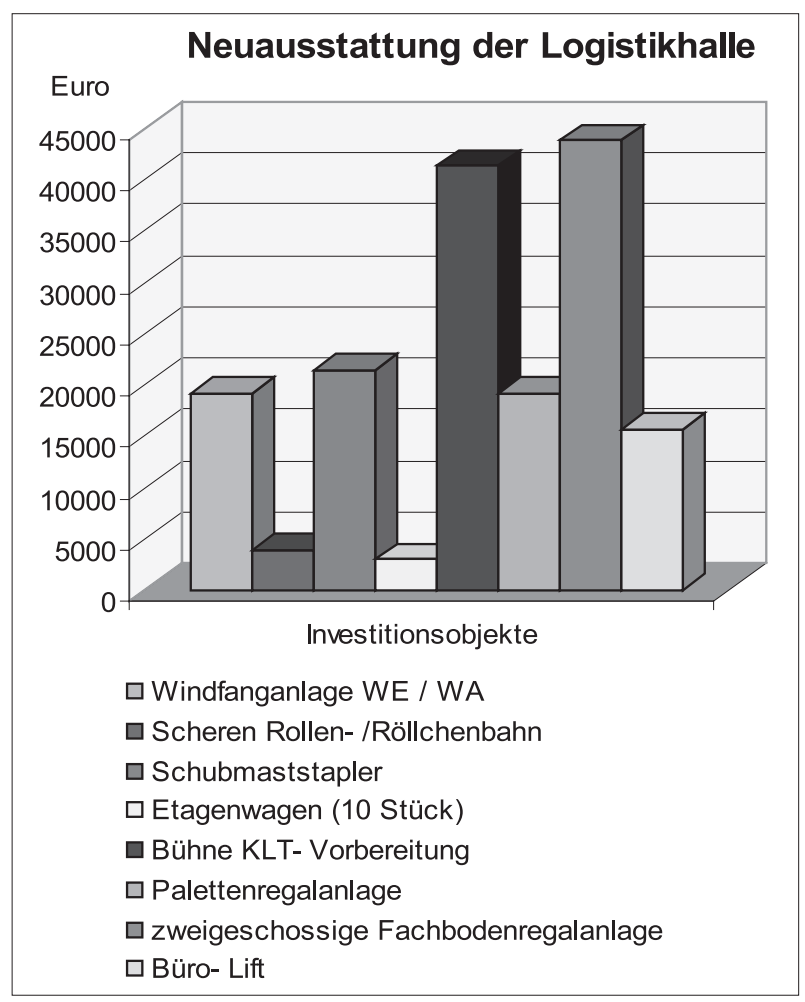

Abbildung 12: Neuausstattung der Logistikhalle

\section{Fazit und Empfehlung}

Bei der Neugestaltung wurden die einzelnen Bereiche in der Halle so angeordnet, dass der Materialfluss einen Art Kreislauf darstellt. Eine Zentralisierung der Lagerbereiche wurde ebenfalls erreicht. Die einzelnen Lagerbereiche befinden sich nun alle in einem Gebäude und sind nebeneinander angeordnet. Die Bevorratung der Artikel erfolgt nach einer durchgeführten $\mathrm{ABC}$-Analyse. Dadurch können Wegstrecken verkürzt werden und die Berücksichtigung der Entnahmehäufigkeit bei der Zuordnung des Lagerplatzes wird sich produktivitätssteigernd auswirken.

Oft zu kommissionierende Artikel werden im Untergeschoss der Fachbodenregalanlage gelagert, Langsamdreher werden im Obergeschoss bevorratet. Oft benötigte Paletten werden in den unteren Ebenen der Reihen 5 und 6 des Palettenregallagers untergebracht. Durch die geringeren Fachhöhen der Fachbodenregale können maximal nur noch 2 Kartons übereinander gelagert werden. Dadurch müssen bei der Kommissionierung nicht mehr so viele Kartons umgestapelt werden und die Kartons werden nicht mehr so gedrückt und Material beschädigt.

Durch eine systematische Nummerierung aller Lagerorte ist die Basis und Voraussetzung für eine effiziente Lagerorganisation geschaffen. Jeder Stellplatz erhält einen logischen und eindeutigen Informationsinhalt. Außerdem wurde eine möglichst enge Anordnung der Arbeitsplätze geschaffen. Die Mechanisierung der Förderwege wurde zwar angestrebt, aber war nicht in allen Bereichen umsetzbar, da die zu beachtenden Grenzen der Wirtschaftlichkeit nicht immer erreicht wurden. Die Transportmittel und Ladeeinheiten wurden gegenseitig angepasst; dabei mussten die Transportmittel auf die Ladeeinheiten zugeschnitten werden. 
Die Ausstattung des Lagerbereiches A mit einer Palettenregalanlage ist die effizienteste Lösung. Da immer die Wirtschaftlichkeit einer Anlage zu beachten ist, sind andere Systeme für die Belange der Funkwerk Dabendorf GmbH nicht geeignet.

\section{Anmerkungen}

[1] www.fwd-online.de

[2] www.ssi-schaefer.de

[3] www.jungheinrich.de

[4] www.ssi-schaefer.de

\section{Literatur}

Arnold, Dieter; „Materialflußlehre“, Verlag Viewig, 2., verbesserte Auflage, 1998

Arnold, D./Isermann, H./Kuhn, A./Tempelmeier, H.; „Handbuch Logistik“, Springer Verlag, VDI, 2., aktualisierte und korrigierte Auflage, 2004

Backmerhoff, Wolfgang; „Beitrag zur Automatisierung von Kommissioniersystemen“, HUSS- VERLAG, 1988

Bernnat, Rainer; „Strategien der Lagerplatzvergabe“ (Rationalisierungspotentiale im Kommissionierlager), Gabler Verlag, 1998

Bichler/Schröter; „Praxisorientierte Logistik“, Kohlhammer, 2., überarbeitete und erweiterte Auflage 2000

Bichler, Klaus/Krohn, Ralf; „Beschaffungs- und Lagerwirtschaft“ (Praxisorientierte Darstellung mit Aufgaben und Lösungen), Verlag Gabler, 8., vollständig überarbeitete Auflage, Oktober 2001

Brockmann/Friemuth/Oster/Sander; „Wie gut ist Ihre Logistik“ (Kennzahlen für Produktionsunternehmen), Verlag TÜV Rheinland, 2. aktualisierte Auflage, 1997

Fischer, W./Dittrich, L.; „Materialfluss und Logistik“ (Potentiale vom Konzept bis zur Detailauslegung), Springer Verlag, VDI, 2. erweiterte Auflage, 2004

Jünemann/Schmidt; „Materialflusssysteme“ (Systemtechnische Grundlagen), Springer-Verlag, 2. Auflage, 2000

Jünemann, R.; „Materialfluss und Logistik“, Springer Verlag Berlin, 1989

Klaus/Krieger; „Gabler-Lexikon Logistik“ (Management logistischer Netzwerke und Flüsse), Gabler Verlag, 1998

Martin, Heinrich; „Transport- und Lagerlogistik“ (Planung, Aufbau und Steuerung von Transport- und Lagersystemen), Viewegs Fachbücher der Technik, 4., überarbeitete und erweiterte Auflage, 2002

Meier-Barthold, Dirk; „Flexibilität in der Material-Logistik“ (Gabler Edition Wissenschaft: Produktion und Logistik), Gabler Verlag, 1999

Olfert, Klaus; „Logistik“, Friedrich Kiehl Verlag, 4., überarbeitete und aktualisierte Auflage, 2003

Rupper, P./Scheuchzer, R. H.; „Lager und Transport Logistik“ (Planung, Steuerung und Kontrolle im Transport- und Lagerbereich), Verlag Industrielle Organisation, 2. Auflage, 1990

Schulte, Christof; „Logistik“ (Wege zur Optimierung des Materialund Informationsflusses), Verlag Franz Vahlen, 3., überarbeitete und erweiterte Auflage, 1999
Tempelmeier, Horst; „Material-Logistik“ (Modelle und Algorithmen für die Produktionsplanung und -steuerung und das Supply Chain Management), Springer Verlag, 4, überarbeitete und erweiterte Auflage, 1999

Vogt, Gerd; „Kommissionier Handbuch“ (Eine Sonderpublikation der Zeitschrift MATERIALFLUSS), Verlag Moderne Industrie AG, 1997

Wannenwetsch, Prof. Dr. Helmut; „Integrierte Materialwirtschaft und Logistik" (Beschaffung, Logistik, Materialwirtschaft und Produktion), Springer Verlag, VDI, 2., überarbeitete Auflage, 2004

\section{Autoren}

Dipl.-Wirtschaftsingenieur (FH) Sven Landvoigt

Funkwerk Dabendorf GmbH

Abteilung Logistik

Märkische Straße

15806 Dabendorf

Tel. +49 3377 316-306

sven.landvoigt@fwd-online.de

Dipl.-Ing. Lutz Pfister, Geschäftsführer

Funkwerk Dabendorf GmbH

Märkische Straße

15806 Dabendorf

Tel. +493377 316-102

lutz.pfister@fwd-online.de

Prof. Dr.-Ing. Herbert Sonntag

Technische Fachhochschule Wildau

Fachbereich Ingenieurwesen/Wirtschaftsingenieurwesen - Logistik

Tel. +493375 508-924

hsonntag@igw.tfh-wildau.de 